\title{
PERCEPÇÃO DO ENFERMEIRO DA ATENÇÃO PRIMÁRIA À SAÚDE FRENTE A
} ATRIBUIÇÃO DE GESTOR DA UNIDADE

\section{NURSES' PERCEPTIONS OF PRIMARY HEALTH CARE VS. UNIT MANAGER ASSIGNMENT}

\section{PERCEPCIONES DE LOS ENFERMEROS DE LA ATENCIÓN PRIMARIA A LA SALUD FRENTE A LA ASIGNACIÓN DE GESTOR LA UNIDAD}

\author{
Hiasmin Marques Cardoso ${ }^{1}$, Grasiele Cristina Lucietto ${ }^{2}$, Rondinele Amaral da Silva ${ }^{3}$, Joely \\ Maria Oliveira ${ }^{4}$, Monique Maira Maciel $^{5}$
}

\begin{abstract}
RESUMO
Objetivo: Compreender as percepções dos profissionais enfermeiros (as) acerca da função de gestor (a) dos serviços e equipes de saúde. Método: Trata-se de um estudo de abordagem qualitativa, tipo descritivo e exploratório. Realizou-se entrevistas por meio de questionários autoaplicáveis no período de setembro a novembro de 2017, com oito enfermeiros (as) gestores (as) atuantes na Atenção Primária a Saúde, especificamente na Estratégia de Saúde da Família em um município do médio norte do Estado de Mato Grosso. Resultados: A análise temática dos contextos resultou na construção de dois eixos temáticos por ordem de relevância, sendo eles: Transição da graduação para o mercado de trabalho e Fatores influenciadores no processo de trabalho do enfermeiro gestor. Conclusão: Através dos achados da pesquisa, verificou-se que os profissionais de enfermagem enfrentam dificuldades na atuação enquanto gestor da atenção primária, processos decorrentes do modelo de formação profissional.

Descritores: Enfermagem; Atenção Primária à Saúde; Administração dos serviços de saúde;
\end{abstract} Liderança.

\begin{abstract}
Objective: To understand the perceptions of nurses professionals about the role of primary health care manager. Method: This is a qualitative study, descriptive and exploratory. Interviews were conducted through self-administered questionnaires from September to November 2017, with eight nurses working in Primary Health Care, specifically in the Family Health Strategy in a municipality in the middle north of the State of Mato Grosso. Results: The thematic analysis of the contexts resulted in the construction of two thematic axes in order of relevance: Transition of graduation to the labor market and Factors influencing the work process of the nurse manager. Conclusion: Through the research findings, it was verified that nursing professionals face difficulties in acting as manager of primary care, processes resulting from the professional training model.

Descriptors: Nursing; Primary Health Care; Administration of health services; Leadership.

\footnotetext{
${ }^{1}$ Graduada em Enfermagem pela Universidade do Estado de Mato Grosso (UNEMAT). Campus Tangará da Serra, Mato Grosso, Brasil.

${ }^{2}$ Enfermeira Mestre em Enfermagem pela Universidade Federal do Estado de Mato Grosso (UFMT). Docente Assistente da Universidade do Estado de Mato Grosso. Campus Tangará da Serra, Mato Grosso, Brasil.

${ }^{3}$ Enfermeiro, Mestre em Ciências Odontológicas Integrada pela Universidade de Cuiabá (UNIC). Docente Auxiliar da Universidade do Estado de Mato Grosso. Campus Tangará da Serra, Mato Grosso, Brasil.

${ }^{4}$ Graduada em Enfermagem pela Universidade Estadual do Mato Grosso - UNEMAT, Mestranda em Saúde Coletiva pela Universidade Federal do Estado de Mato Grosso (UFMT), Brasil.

${ }^{5}$ Enfermeira Especialista. Docente Auxiliar da Universidade do Estado de Mato Grosso. Campus Tangará da Serra, Mato Grosso, Brasil.
} 


\section{RESUMEN}

Objetivo: Comprender las percepciones de los profesionales enfermeros acerca de la función de gestor de la Atención Primaria a la Salud. Método: Se trata de un estudio de enfoque cualitativo, tipo descriptivo y exploratorio. Se realizaron entrevistas a través de cuestionarios autoaplicables en el período de septiembre a noviembre de 2017, con ocho enfermeros (as) gestores (as) actuantes en la Atención Primaria a la Salud, específicamente en las Estrategias de Salud de la Familia en un municipio del medio norte del Estado de Mato Grosso. Resultados: El análisis temático de los contextos resultó en la construcción de dos ejes temáticos por orden de relevancia, siendo ellos: Transición de la graduación al mercado de trabajo y Factores influyentes en el proceso de trabajo del enfermero gestor. Conclusión: A través de los hallazgos de la investigación, se verificó que los profesionales de enfermería enfrentan dificultades en la actuación como gestor de la atención primaria, procesos derivados del modelo de formación profesional.

Descriptores: Enfermería; Atención Primaria a la Salud; Administración de los servicios de salud; Liderazgo.

\section{INTRODUÇÃO}

No contexto histórico, Florence Nightingale por volta do século XIX durante a Guerra da Criméia, iniciou à instituição do gerenciamento em enfermagem, em razão da necessidade de organizar e administrar os hospitais de Scutari na Turquia para o recebimento da maior parte dos feridos durante a guerra. ${ }^{1}$

No Brasil, a formação de profissionais graduados em enfermagem é realizada por mais de 880 instituições sob a orientação das Leis de Diretrizes e Bases da Educação Brasileira - LDB, e das Diretrizes Curriculares Nacionais - DCN, para os Cursos da Área da Saúde. Portanto, configura-se como potencialmente capaz de influir na construção de novas práticas, entre elas aquelas relacionadas ao gerenciamento e administração. $^{2}$

Com o advento da reforma sanitária e construção do Sistema Único de Saúde (SUS) surgiram novas demandas assistenciais e gerenciais, essa condição assume significado estratégico para a consolidação de políticas públicas e reorganização dos modelos de saúde. No Brasil, a Atenção Primária a Saúde incorpora os princípios do SUS, adota a designação para a Estratégias de Saúde da Família, e enfatiza a reorientação do modelo assistencial através do sistema universal e integrado. A Atenção Primária a Saúde apresenta a estratégia de organização da atenção à saúde para suprir as necessidades de forma regionalizada, descentralizada, contínua, sistematizada para integrar ações de promoção, prevenção da saúde e reabilitação. ${ }^{3-4}$

Para que as atribuições especificas nos níveis de Atenção Primária a Saúde e Estratégia de Saúde da Família seja efetuada com sucesso, torna-se necessário a atuação de uma equipe multidisciplinar, composta basicamente por médicos generalista, enfermeiro e técnicos em 
enfermagem, agentes comunitários de saúde e recepcionistas. Sendo que destes profissionais da equipe multidisciplinar, o enfermeiro atua na liderança, nas funções de gerenciamento da assistência, gestão da equipe e da unidade. ${ }^{4}$

A Resolução CNE/CES No 3, de 7 de novembro de 2001, institui diretrizes curriculares nacionais do curso de graduação em Enfermagem, estabelece competências e habilidades gerais que todo enfermeiro deve dispor, dentre elas: a capacidade de tomar decisões, habilidades de comunicação, habilidade para assumir posições de liderança, aptidão para tomar iniciativa, fazer $\mathrm{o}$ gerenciamento $\mathrm{e}$ administração tanto da força de trabalho quanto dos recursos físicos e materiais e de informação. ${ }^{5}$

A Lei $n^{\circ} 7.498$ de 25 de junho de 1986, no Artigo 11, relata ser de competência do enfermeiro chefiar o serviço e a unidade de Enfermagem em instituição pública e privada; organizar e dirigir os serviços de Enfermagem e suas atividades técnicas e auxiliares; planejar, organizar, coordenar, executar e avaliar os serviços de assistência de Enfermagem. Tem-se o enfermeiro como líder da equipe de enfermagem, a atribuição provém da posição hierárquica adquirida durante $o$ período de formação embasada nos conhecimentos técnico e científico. ${ }^{6}$
Percebe-se que, há enfermeiros que realizam suas atividades com bom desempenho assistencial, porém apresentam fragilidades no desenvolvimento das atribuições administrativas ou o inverso, o que evidencia uma dificuldade em integrar as dimensões gerenciais e assistenciais, e assim prevalece a fragmentação da assistência. O profissional enfermeiro tende a menosprezar a atividade a qual não desenvolve, e consequentemente, valorizar a função que desempenha, sendo ela gerencial ou assistencial. $^{7}$

O encargo da tomada de decisões e da administração das equipes com o objetivo de mantê-las satisfeitas e produtivas, é do enfermeiro. Anseia-se que o enfermeiro desempenhe função de mediador de conflitos não apenas entre a própria equipe, mas sim em todo contexto interdisciplinar, caracterizando uma complexa atividade gerencial. ${ }^{8}$

O gerenciamento em enfermagem é um processo que exige especificidade, objetividade e finalidade, enfatiza-se a organização do trabalho a partir dos instrumentos e recursos administrativos que buscam uma assistência de qualidade e através de uma equipe de enfermagem qualificada, em que o enfermeiro possa estar no domínio das atividades gerenciais, articulando o processo gerencial com o assistencial. Infere-se ainda que, após o 
profissional deixar a vida acadêmica passa a lidar diretamente com os problemas relacionados a assistência técnica e gerenciamento de equipe e a primeira experiência profissional pode acarretar em grandes obstáculos e frustações. ${ }^{9}$

Compreender as dificuldades enfrentadas pelos enfermeiros que desempenham a função de gestor é indispensável, visto que os aspectos gerenciais têm relação direta com a qualidade dos serviços prestados à população. Portanto, justifica-se a realização do estudo, uma vez que o profissional enfermeiro possui atribuições de gestor tanto dos serviços assistenciais quanto gerencias da equipe multidisciplinar, atuar como facilitador para mudanças de paradigma do modelo de saúde, o biomedicista centrado na doença, e atuar como agente transformador do cuidado, agindo de forma integral a fim de beneficiar a saúde da coletividade em todas as fases de promoção da saúde, prevenção, recuperação e reabilitação da saúde. Outro fator importante, é demonstrar que apesar de árdua as atribuições dos enfermeiros nas funções de gerenciar, coordenar e assistir, são peças chave para uma assistência qualificada pautadas nos princípios doutrinários do Sistema Único de Saúde. Diante disso, objetivo do estudo é compreender as percepções dos profissionais enfermeiros acerca da função de gestor da Atenção Primária a Saúde, em especial os atuantes na Estratégia de Saúde da Família.

\section{MÉTODO}

Trata-se de estudo descritivo, exploratório, de abordagem qualitativa. A abordagem qualitativa permite compreensão da realidade social, uma vez que trabalha com as crenças, os valores e atitudes. ${ }^{10}$

A investigação foi desenvolvida em um município da região médio norte matogrossense. O serviço de atenção primária à saúde do munícipio conta com 21 Estratégia de Saúde da Família presentes no Cadastro Nacional de Estabelecimento de Saúde (CNES). A pesquisa obteve como cenário, oito (08) Estratégias de Saúde da Família e oito (08) enfermeiros das respectivas unidades, sendo estes selecionados de forma aleatória.

A seleção dos partícipes se deu através dos critérios de inclusão e exclusão. Foram incluídos enfermeiros atuantes na área de gerenciamento da equipe da Estratégia Saúde da Família no período superior de seis meses, a delimitação desse tempo se deve a necessidade de o profissional ter vivenciado um período de experiência, e assimilar através da prática aspectos relacionados ao gerenciamento. Foram excluídos os profissionais que estavam de licença, atestado médico ou 
férias, e/ou outros profissionais afastados ou aposentados da profissão, pois dificultaria a obtenção do contato e acesso aos mesmos, correndo o risco de retardar o processo de coleta de dados.

A coleta de dados ocorreu no período de setembro a novembro de 2017. Os instrumentos de pesquisas foram compostos por questionários semiestruturado, continham dez questionamentos, sendo a primeira questão referente a informações do perfil sócio demográfico dos profissionais enfermeiros: Sexo, idade, cor autodeclarada e estado civil. As demais questões eram específicas sobre as atividades gerenciais, potencialidades e limitações no desenvolvimento das atribuições de gestor da unidade. $\mathrm{O}$ instrumento foi confeccionado pelos autores e realizado teste piloto para sanar possíveis equívocos oriundos da construção, sendo este aprovado pelo Comitê de Ética em Pesquisa (CEP).

No período inicial a coleta de dados, realizou-se agendamento e contato prévio com a coordenação da Estratégia de Saúde da Família, para apresentação sobre a pesquisa e autorização do responsável superior para execução da mesma. Após autorização por parte da coordenação, efetuou-se o primeiro contato por telefone com os profissionais de enfermagem das estratégias de saúde da família, agendando assim um encontro. No segundo momento, realizou - se a apresentação do Termo de Consentimento Livre e Esclarecido (TCLE), para oportunizar a esses profissionais a compreensão do objetivo e a importância do estudo. Em seguida, após as assinaturas do TCLE, foram entregues os questionários autoaplicáveis e devolvidos aos pesquisadores conforme os profissionais finalizavam o preenchimento. O período de coleta de dados foi encerrado a partir do momento em que as narrativas passaram a apresentar a exaustividade das informações de interesse, tal como proposto pela saturação dos dados. ${ }^{10}$

Após a coleta dos dados, realizou-se a organização das informações na íntegra de forma minuciosa, para a construção e revisão da análise. Efetuou-se a análise descritiva de todas as variáveis do estudo, através do método de digitação, programa Windows 10.0. Em seguida, os dados foram agrupados em categorias analíticas, a partir dos sentidos e dos significados das narrativas dos participantes.

O material empírico foi analisado e categorizado a luz da análise de conteúdo do tipo temática, ou seja, diz respeito a técnicas de pesquisa que admitem tornar replicáveis e válidas inferências sobre dados de uma determinada situação, através de procedimentos especializados e científicos. $^{11}$ 
Todas as fases de pesquisa contemplaram a Resolução n466, de 12 de dezembro de 2012 do Conselho Nacional de Pesquisa em Saúde. Aprovado pelo Comitê de Ética em Pesquisa (CEP) através do parecer número 082614/2017. Os dados que compuseram este estudo mantiveram-se confidenciais, garantindo o sigilo de participação durante toda pesquisa, inclusive na divulgação da mesma. Para garantia do anonimato, os nomes foram substituídos por letras e números (Participante: enf.1, enf.2, enf.3).

\section{RESULTADOS}

Participaram deste estudo 08 profissionais atuantes na Estratégia de Saúde da Família, sendo sete (07) do sexo feminino e um (01) sexo masculino, a faixa etária variou entre 28 e 30 anos e o tempo de serviço de (04) anos e nove (09) anos. Em relação a estabilidade profissional, um (01) dos profissionais é efetivo pelo município e os demais participantes possuem cargo ocupados por meio de processos seletivos por tempo determinado.

A análise temática dos contextos resultou na construção de dois eixos temáticos por ordem de relevância, sendo eles: Transição da graduação para o mercado de trabalho e; Fatores influenciadores no processo de trabalho do enfermeiro gestor.

\section{Transição da graduação para o mercado de trabalho}

Enfatiza as dificuldades enfrentadas na vivência do primeiro emprego, o gerenciamento dos conflitos no ambiente de trabalho; as expectativas do primeiro emprego; necessidade de aperfeiçoamento na área da gestão; dificuldades em lidar com o sentimento de despreparo profissional. Os profissionais responderam sob a ótica de suas trajetórias profissionais e ressaltaram que as expectativas, o medo e a insegurança estiveram presentes no início da carreira de todos eles.

As narrativas inferem que este despreparo possa ser um reflexo da formação profissional, conforme relatos, os cursos de graduação em enfermagem têm oferecido pouco conteúdo relacionado ao papel do enfermeiro como gestor e liderança, e sim com abordagem para um olhar assistencial.

Sim, há insegurança nas atividades, a falta de mais aulas gerenciais. Enf.4

Dificuldades em compreender de início que nem tudo depende da nossa vontade de resolver algum problema, trabalhar como gestor de serviços, principalmente no SUS, requer paciência, jogo de cintura e saber gerenciar problemas, tais atitudes pouco desenvolvidas na graduação já que não 
podemos interferir nos campos de estágios, em sua maioria só observar. Enf.5

O primeiro emprego é sempre difícil, devido os medos, insegurança e também porque em relação à gestão a graduação não forneceu muita experiência prática. Enf.7

Quando questionados sobre as expectativas referente às atribuições e funções do enfermeiro na gestão, especificamente relacionada a experiência do primeiro emprego, todos os profissionais entrevistados relataram que suas expectativas e anseios eram frutos da falta de conhecimento e experiências, e que essas estão diretamente ligadas à complexidade das atividades gerenciais atribuídas ao enfermeiro na sua rotina. As narrativas a seguir demonstram algumas dessas percepções:

Confesso que me decepcionei, pois, muitas atividades gerenciais não dependem somente de mim e sim de um conjunto de colaboradores e setores que estejam dispostos também em serem resolutivos. Enf. 4

Não tinha tantas expectativas e sim anseios, como o medo de não conseguir gerir o serviço de saúde ao qual eu fui destinada. Saímos da faculdade com a sensação de que não sabemos nada e que não seremos capazes, ao nos depararmos na função de gestor vamos aos poucos adaptando com a função e percebendo que muito do que aprendemos na graduação mesmo que apenas na teoria nos ajuda muito.

Enf. 5

Ao serem questionados sobre as perspectivas que nutriam as prerrogativas do enfermeiro gestor, ainda quando os mesmos ingressaram e atuavam pela primeira vez, os profissionais responderam e ressaltaram que as expectativas eram amplas, algumas não tão positivas quanto outras, porém ainda enfrentam dificuldades no papel de gestores.

As minhas expectativas eram boas, depois durante o início dos trabalhos e por uns cinco anos essas expectativas foram surpreendidas e superadas e há 4 anos estas expectativas estão ruins. Ando muito desmotivada com a Saúde Pública no Brasil. Enf.4

Minhas expectativas continuam as mesmas de quando iniciei no meu primeiro emprego como enfermeira. Continuo buscando dar o meu melhor na minha função de gestora. Enf. 5

$A$ realidade é que o enfermeiro deveria exercer a função de gestor unicamente e não juntamente com a função assistencial e hoje continuo com a mesma visão. 
Enf.7

Com relação aos aprendizados adquiridos com a responsabilidade de exercer as atividades de um gestor, os relatos apontam o quão é fundamental vivenciar a prática, ter conhecimento das Políticas Públicas de Saúde e da burocracia, possuir agilidade na execução das atribuições, traçar estratégias, planejamento e levantamento dos problemas para o alcance de metas para a obtenção da melhora dos serviços prestados, além de tudo, o profissional precisa ser um bom líder, ir em busca de conhecimentos, principalmente nas especialidades de gestão em enfermagem.

A experiência nos faz entender a importância que a burocracia tem no setor onde trabalhei e trabalho e também as dificuldades que ela traz, e que ações mal planejadas refletem por muitos e muitos anos nas equipes de saúde e na população. Enf1

Estou me capacitando ainda, terminando uma pós, espero me aperfeiçoar a cada dia. Enf3

\section{Fatores influenciadores no} processo de trabalho do enfermeiro gestor

Aborda fatores existes no cotidiano dos profissionais que interferem no processo de trabalho gerencial, estes foram destacados por ordem e relevância, sendo eles: Motivação e/ou desmotivação no ambiente de trabalho; o trabalhar em equipe; necessidade de traçar estratégias e ferramentas para gerir com qualidade; atividades burocráticas e divisão do tempo entre as atividades assistenciais e gerenciais.

O fator motivação e/ou desmotivação foi um dos aspectos relatados pelos partícipes da pesquisa, com divergência nos relatos, pois alguns disseram sentir-se motivados, realizados, agradecidos por desempenhar a função de gerência, em contrapartida, outros relatos demonstraramse insatisfeitos, desmotivados e até mesmo obrigados a exercer a função de gestor.

O que me motiva é o fato de ver como as pessoas, tanto os pacientes, quanto os profissionais de saúde, são beneficiados através da minha gestão nos serviços de saúde, nos quais já atuei. Enf.1

Não gosto de ser gestora, trabalho porque não tive opção, prefiro ser enfermeiro assistencial. Enf.2

Outro fator influenciador é a importância do enfermeiro gestor para a equipe, essa pode se apresentar tanto positiva quanto negativamente.

Sim. A equipe observa muito o gestor, mesmo que seja em um processo lento, podemos influenciar motivar a equipe. 
Enf.4

Sim. Porque o gestor é capaz de mover a sua equipe com positividade ou negatividade, depende muito da sua capacidade de liderar. Enf. 3

Os profissionais visam buscar além da gestão democrática, métodos e estratégias de liderança para que possam se posicionar diante da equipe como um líder, frente as dificuldades burocráticas.

Sempre realizo gestão compartilhada, com a característica de colocar o cuidado e as necessidades do usuário em primeiro lugar, esse compartilhamento acontece entre a equipe e o próprio paciente. Enf.1

Procuro realizar reuniões semanais com a equipe, na qual evito chamar a atenção de alguma pessoa especifica. Procuro envolver todos os membros da equipe na gestão do serviço de saúde. Enf5

Burocracia, falta de pessoas $e$ também capacitação, o tempo não exclusivo para a gestão o que não conseguimos é ter o controle da evolução dos processos e metas e atender ao público com técnicas com a mesma qualidade. Enf. 1

Atingir os objetivos para alcançar uma gestão de sucesso torna-se uma tarefa árdua para os profissionais de enfermagem frente as dificuldades presentes no dia-a-dia do gestor. As dificuldades são descritas como fatores influenciadores no processo de trabalho de gerência do enfermeiro, destaca-se os processos burocráticos considerada um grande desafio e a divisão do tempo entre as funções gerenciais e assistenciais.

\section{DISCUSSÃO}

O eixo temático transição da graduação para o mercado de trabalho reforça o quanto é difícil o processo de adaptação nas atividades gerenciais, uma vez que, os profissionais enfermeiros não são preparados suficientemente no período de formação para lidar com a gestão e as suas especificidades. O primeiro emprego é encarado como algo desafiador e é visto como uma ameaça, pois haverá novas necessidades, tanto comportamentais quanto de competência e aptidão para os profissionais recém-graduados em Enfermagem. ${ }^{11}$

A pressão dos serviços de saúde sobre os enfermeiros recém-formados é notável, especialmente por ocuparem cargos de chefias, liderar as equipes de enfermagem que possui maior experiência e habilidade técnica. Diante desses aspectos, os profissionais enfermeiros sentem-se inseguros e insuficientemente preparados para enfrentar tal realidade. $^{12}$ 
Nesse contexto, torna-se necessário indagar o cenário das práticas gerenciais em face do Projeto Político Pedagógico dos cursos de graduação em Enfermagem, pois o modelo de ensino ainda é centrado no assistencialismo com visão hospitalocêntrica, biomedicista e curativa. Logo, há necessidade de traçar estratégias durante a graduação, mudanças nos Planos Pedagógicos para que se envolva o docente, o aluno e o profissional dos serviços de saúde, inquirindo as demandas gestoras ao processo de trabalho, tendo como foco a análise das competências gerenciais dos acadêmicos durante a graduação e estágios curriculares que viabilizem aos acadêmicos discernir suas limitações e potencialidades referente as atribuições gerenciais. ${ }^{11}$

Diante das atribuições dos enfermeiros, discutidas isoladamente entre os planos da educação e trabalho, as competências dos enfermeiros, tornaram-se um reflexo das necessidades do mercado, implicam em profissionais competentes e conscientes de seu papel gerencial. Dessa forma, tem se a necessidade de adaptação dos profissionais a certa instabilidade de suas atuações, definidas e formadas pela prática das instituições de ensino e de serviços. ${ }^{2,11}$

Além das necessidades de modificação no ensino da formação, tem-se processos de trabalho na saúde, desenvolvidos através de relações hierárquicas, com enfoque nas individualidades profissionais, o que se faz ressaltar suas fragilidades. Faz-se necessário reconhecer as fragilidades dos serviços de forma integral, os processos de trabalhos se apresentam de forma fragmentada, com saberes isolados e individuais, acarretando dificuldades para os serviços de gestão. ${ }^{12}$

O enfermeiro precisa desdobrar conhecimentos e habilidades acerca dos instrumentos próprios do gerenciamento da assistência de enfermagem, bem como desenvolver competências que culminem em melhorias nos âmbitos assistencial e gerencial. ${ }^{13}$

A falta de conhecimento das atribuições do gerente ou líder acaba limitando alguns profissionais, consequentemente, isso reflete na assistência, ressaltado a importância da gestão de qualidade o que se considera um indicar de qualidade dos serviços. ${ }^{14}$

No que se refere as expectativas dos enfermeiros sobre as competências gerenciais, percebe-se que, de forma geral, as avaliações destes foram positivas em relação as perspectivas, uma vez que desenvolveram competências e habilidades ao decorrer da vida profissional. Ao mesmo tempo, o perídio de experiência e vivências auxiliam no desenvolvimento de habilidades e competências gerenciais, a modificação de suas expectativas pode 
ocorrer negativamente em alguns casos, como evidenciado nas narrativas de participantes que se demostraram desmotivados na realização do trabalho como gestor.

Nesta perspectiva, espera-se que o enfermeiro seja capaz de executar seu papel de gestor, visto que as atividades assistenciais e gerenciais não devem ser executadas de maneira dissociada. Em meio a incandescência dos processos formativos e de atenção à saúde, evidencia-se nesse contexto a necessidade de mudanças, considerando a gestão como um potencial mais amplo, em que engloba a formação de profissionais também para a liderança e gerenciamento, como uma repartição do mercado. Verifica-se a necessidade de ter atributos específicos do enfermeiro gestor, além do reconhecimento da importância e efetivação do trabalho em equipe. Neste modelo assistencial, é imprescindível a busca por um perfil mínimo necessário para uma atuação de qualidade, com conhecimentos específicos para executar as atividades propostas para esse cenário.

O eixo temático fatores influenciadores no processo de trabalho do enfermeiro gestor aborda sobre as dificuldades enfrentadas no cotidiano dos profissionais que interferem no processo de trabalho gerencial, como a motivação e/ou desmotivação no ambiente de trabalho. A desmotivação dos profissionais pode ocasionar a desestruturação nos serviços de enfermagem, o que pode originar a desvalorização dos profissionais, afetando diretamente no trabalho do enfermeiro. ${ }^{15} \mathrm{O}$ líder tem papel fundamental para propiciar e conservar clima harmônico e motivador no ambiente de trabalho, deverá fornecer meios para que os profissionais da equipe se sintam encorajados e engajados para desempenhar as funções diárias, consequentemente a obtenção de resultados positivos para o serviço. ${ }^{16-}$ 17

Diante dos aspectos de desmotivação, nota-se a necessidade de traçar estratégias e ferramentas, estas foram observadas como fatores influenciadores no processo de gestão do enfermeiro, como por exemplo: a importância de manter a interação com a equipe, como a realização das reuniões; proporcionar e manter o diálogo para compartilhar ações e metas; realizar distribuições de funções e atribuição para cada indivíduo que constitui a equipe. Sendo assim, o processo de planejar conta com o uso da motivação e encorajamento de todos para o alcance das metas. ${ }^{17}$

O estilo de liderança que mais estão presentes entre os profissionais de enfermagem é o democrático. Porém, nesse âmbito, a enfermagem ainda enfrenta 
diversos desafios na liderança, devido ao trabalho fundamentar-se na assistência com divisão técnica. A liderança pautada no diálogo, o saber ouvir, ter comprometimento e estimular o trabalho em equipe, são comportamentos vistos como instrumentos positivos para uma liderança de sucesso. É importante que o enfermeiro consiga identificar o processo de liderança, e que por meio dele, permite guiar a equipe para trabalhar entusiasmada, e para tanto, deve propor estratégias motivadoras a execução das tarefas a fim de atingir os objetivos de maneira coletiva. ${ }^{7,18-}$ 19

O enfermeiro é o responsável pela tomada de decisões sobre os serviços prestados, logo faz-se necessário a obtenção dos conhecimentos e habilidade para desempenhar as funções de gestão diante dos desafios enfrentados, como o despreparo profissional em decorrência do modelo de formação, pautado no modelo hospitalocêntrico e fragilizado nos aspectos gerenciais; a hierarquização dos serviços; aspectos burocráticos das políticas públicas dos serviços de saúde; administração do processo de trabalho; necessidade de cumprir metas esperadas pelo Ministério da Saúde; pressão psicológica; lidar com a saúde da coletividade, pois a APS é a porta de entrada do usuário para os demais serviços de saúde; lidar com a falta de recursos e materiais essenciais para a prestação de serviços; a supervisão da equipe multidisciplinar; o surgimento de sentimento de frustração, descontentamento nos profissionais ocasionado sentimento de desmotivação e a fragmentação das atribuições gerenciais e assistenciais. ${ }^{12,17,20}$

Nesse sentindo, o profissional enfermeiro precisa estar capacitado para lidar com as atribuições de gestor em nível de Atenção Primária, em decorrência das características e peculiaridades que este nível oferece, por se tratar da porta de entrada do usuário nos demais serviços de saúde, e principalmente por ser o campo onde permite atuar nos níveis da promoção da saúde, prevenção de agravos, recuperação e reabilitação.

\section{CONCLUSÃO}

O estudo possibilitou conhecer as percepções dos profissionais enfermeiros em sua vivência profissional acerca da função de gestor dos serviços na Atenção Primária a Saúde, em especial dos enfermeiros atuantes na Estratégia de Saúde da Família. Através dos achados da pesquisa, verificou-se que os profissionais de enfermagem enfrentam percalços na atuação enquanto gestor em decorrência da formação, ainda pautado no modelo hospitalocêntrico, assistencialista e fragilizado nos aspectos gerenciais. 
Os participantes do estudo passam por dificuldades que envolvem a falta de conhecimento específico sobre gestão e liderança das equipes; desmotivação da equipe, sobrecarga de trabalho e grande demanda de atividades assistenciais. Relataram a dificuldade em se participar de estudos continuados sobre gestão, por falta de oportunidades e condições desfavoráveis de trabalho. Foi predominante o desinteresse sobre a área em questão, o que reflete na situação do mercado de trabalho, onde tornar-se necessária a presença de profissionais capacitados para a gestão.

Verificou-se que, as dificuldades descritas nos achados estão relacionadas ao processo de formação durante a academia, influenciados por grades curriculares que possuem o olhar assistencial, deixando as questões gerenciais e de liderança com menor ênfase. A postura adotada pelas instituições formadoras resulta em dificuldade futuras aos profissionais de enfermagem, que necessitam de conhecimento e experiências para gerenciar os serviços de saúde com qualidade. Observou-se que estes profissionais enfrentam diversos desafios estes procuram adotar estratégias e ferramentas para gerir com qualidade, lidar com as atividades burocráticas e divisão do tempo entre as atividades assistenciais e gerenciais.
Portanto, o estudo reafirma a existência de uma conjuntura desfavorável para o fortalecimento do modelo de gestão dos profissionais, relacionadas aos conhecimentos gerenciais que envolvem a capacidade de liderança da equipe e o pouco conhecimento burocrático dos serviços para o maior controle e qualidade. Com isso o estudo fornece informações e contribuições no âmbito das pesquisas, uma vez que, área de enfermagem está diretamente relacionada com o processo de gestão, porém ainda é um grande desafio para os profissionais executar atribuições e competências gerenciais. Essa reflexão mostra a fragilidade existente na Enfermagem Brasileira, visto que é necessário remodelar o cenário de ensino e ajustar conforme as necessidades do mercado de trabalho.

O estudo também levanta questionamentos e reflexões para os gestores e administradores dos serviços da Atenção Primária a Saúde, para que estes possam contribuir e ser atuantes ativos com ações que visem a redução das fragilidades abordadas, como sugestões, fazer mais investimentos nas práticas de educação continuada, a fim de aperfeiçoar e atualizar os conhecimentos, além de tudo torna-se essencial proporcionar condições favoráveis de trabalho para que estes sintam-se motivados em sua profissão, e consequentemente refletir positivamente na 
qualidade dos serviços prestados. Diante disso, se fazem necessários a realização de novos estudos, análises da grade curricular e metodologias de ensinos das graduações de enfermagem, para que possam ir de acordo com as necessidades dos serviços de saúde, assim como fortalecer as parcerias e articulações entre as instituições de ensino superior juntamente a gestão e coordenação dos serviços de saúde local.

\section{AGRADECIMENTOS}

Agradecimentos a todos os sujeitos participantes da pesquisa, pois foram imprescindíveis para a construção deste estudo.

\section{REFERÊNCIAS}

1-Santana IF, Silva JP. Gerenciamento em enfermagem: os empecilhos e benefícios encontrados na prática de gerenciamento de enfermagem de um hospital público. Rev FAESF. 2018; 2(2):45-56.

2- Teixeira E, Fernandes JD, Andrade AC, Silva KL, Rocha MEMO, Lima RJO.

Panorama dos cursos de graduação em enfermagem no Brasil na década das diretrizes curriculares nacionais. Rev Bras Enferm. 2013; 66(N Esp):102-10.

3- Merhy EE. Um dos grandes desafios para os gestores do SUS: apostar em novos modos de fabricar modelos de atenção. In: Merhy EE, Magalhães Júnior HM, Rimoli J, Franco TB, Bueno WS, et al. O trabalho em saúde: olhando e experienciando o SUS no cotidiano. São Paulo: Hucitec; 2007. p. 15-35.

4- Matta GC, Morosini MVG. Atenção primária à saúde. In: Escola politécnica de saúde Joaquim Venâncio, Estação de trabalho observatório de técnicos em saúde, organizadores. Dicionário da educação profissional em saúde. Rio de Janeiro: Fiocruz; 2009. v. 2 p. 23-25. 5- Conselho Nacional de Educação (Brasil). Resolução CNE/CES n ${ }^{\circ}$ 3, de 7 de novembro de 2001. Institui Diretrizes Curriculares Nacionais do curso de graduação em enfermagem [Internet]. Brasília, DF: CNE; 2001 [citado em 24 jan 2019]. Disponível em: http://portal.mec.gov.br/cne/arquivos/pdf/C ES03.pdf

6- Conselho Federal de Enfermagem (Brasil). Lei Cofen n ${ }^{\circ}$ 7.498/86.

Regulamentação do exercício de enfermagem. Brasília, DF: Conselho Federal de Enfermagem; 1986.

7- Souza FA, Paiano M. Desafios e dificuldades enfrentadas pelos profissionais de enfermagem em início de carreira. REME Rev Min Enferm. 2011; 15(2):267-73.

8- Marta CB, Lacerda AC, Carvalho ACS, Stipp MAC, Leite JL. Gestão de conflitos: competência gerencial do enfermeiro. Rev Pesqui Cuid Fundam. [Internet]. 2010 [cited 2019 jan 24]; 2(Ed Supl):604-8. Disponível em:

http://www.seer.unirio.br/index.php/cuidad ofundamental/article/view/1062/pdf_228 9- Lima RS, Dázio EMR, Rosado SR, Lourenço EB. Dificuldades e facilidades no gerenciamento de enfermagem no hospital na perspectiva do enfermeiro. Rev Enferm UFPE on line [Internet] 2014 [citado em 24 jan 2019]; 8(12):4253-60. Disponível em: https://periodicos.ufpe.br/revistas/revistaen fermagem/article/view/10171/10706 10- Minayo MCS. O desafio do conhecimento: pesquisa qualitativa em saúde. 14ed. São Paulo: Hucitec; 2015. 11- Guerra KJ, Spiri WC. Compreendendo o significado da liderança para o aluno de graduação em enfermagem: uma abordagem fenomenológica. Rev Bras Enferm. 2013; 66(3):399-405. 12- Martins AB, Cardoso ML. Uma reflexão crítica sobre as dificuldades do enfermeiro recém-formado no exercício da 
liderança. Vitrine Prod Acad. 2015; 3(2): 144-52.

13- Santos AM, Nóbrega IKS, Assis MMA, Jesus SR, Kochergin CN, Bispo Júnior JP, et al. Desafios à gestão do trabalho e educação permanente em saúde para a produção do cuidado na estratégia saúde da família. Rev APS. 2015; 18(1):39-49. 14- Sade PMC, Peres AM. Development of nursing management competencies: guidelines for continuous education services. Rev Esc Enferm USP. 2015; 49(6):988-94. 15- Santos MCL, Braga VAB, Fernandes AFC. Nível de satisfação dos enfermeiros com seu trabalho. Rev Enferm UERJ. 2016; 6(1):101-5.

16- Lanzoni GMM, Magalhães ALP, Costa VT, Erdmann AL, Andrades SR, Meirelles BHS. Becoming nursing manager in the nested and complex border of caring and management dimensions. Rev Eletrônica Enf. [Internet]. 2015[citado em 24 jan 2019]; 17(2):322-32. Disponível em: https://www.fen.ufg.br/revista/v17/n2/pdf/ v17n2a16.pdf

17- Neves VR, Sanna MC. Concepts and practices of teaching and exercise of leadership in nursing. Rev Bras Enferm. 2016; 69 (4):686-93.

18- Ruffatto J, Pauli J, Ferrão AR. Leadership style influence on the motivation and conflict inter-personal in family companies. Rev Adm FACES Journal. 2017; 16(1):29-44.

19- Paula GF, Figueiredo ML, Camargo

FC, Iwamoto HH, Caixeta CRCB.

Concepções de liderança entre enfermeiros assistenciais de um hospital do Norte de Minas Gerais. Rev Eletrônica Enferm. [Internet]. 2012[citado em 24 jan 2019]; 14(4):821-30. Disponível em: https://revistas.ufg.br/fen/article/view/1510 2/13346

20- Lima FS, Amestoy SC, Jacondino MB, Trindade LL, Silva CN, Júnior PRBF. The exercise of leadership of nurses in the family health strategy. Rev Pesqui Cuid Fundam. [Internet]. 2016[citado em 24 jan
2019]; 8 (1):3893- 906. Disponível em: http://www.seer.unirio.br/index.php/cuidad ofundamental/article/view/3905

RECEBIDO: 03/03/2019

APROVADO: 07/07/2019

PUBLICADO: $12 / 2019$ 